\title{
The diagnostic value of faecal trypsin estimation in chronic pancreatic disease
}

\author{
G. K. MCGOWAN AND M. R. WILLS \\ From the Department of Pathology, United Bristol Hospitals
}

SYNOPSIS Faecal trypsin levels have been estimated by a photometric azo-casein method in 242 children and 45 adults. Analysis of the results shows that this is not a specific test of pancreatic dysfunction but that it appears to be a useful screening test for the selection of patients who may have severe pancreatic disease and on whom further specific investigations should be performed.

As specific evidence of pancreatic dysfunction cannot easily be obtained by clinical or radiological examination, the diagnosis of pancreatic disease is largely dependent on laboratory procedures.

Pancreatic dysfunction may be associated with an increase of pancreatic enzymes in the blood, with evidence of diminished secretion of pancreatic enzymes into the gut, or with abnormal carbohydrate tolerance. Estimations of pancreatic enzymes in the blood are of value only in the diagnosis of acute or relapsing pancreatitis, and are of no value in the diagnosis of chronic pancreatic diseases when the gland is atrophic and fibrosed. Evidence of diminished secretion of pancreatic enzymes into the gut can be sought by estimation of tryptic activity in duodenal juice and faeces, and by examination of faeces for undigested and unabsorbed food. Of these, examination of duodenal juice is the most valuable, but requires intubation and is hardly suitable for a routine screening test. Evidence of food remnants in faeces is non-specific, as other conditions such as diarrhoea or steatorrhoea may cause impaired digestion or absorption; an increase in unsplit fat, which is highly suggestive of pancreatic insufficiency, is by no means always present. Similarly, reduced carbohydrate tolerance is not even specific for disease of the Islets of Langerhans, still less of the whole gland, though its demonstration is useful confirmation of pancreatic disease.

This paper is concerned particularly with the value of faecal trypsin estimations in the diagnosis of chronic disorders of the pancreas.

The estimation of tryptic activity is based on the splitting of a suitable protein substrate. The first method to be used routinely for clinical purposes was that of Andersen and Early (1942), in which Received for publication 30 May 1961. doubling dilutions of the sample are made and the highest dilution which liquefies tubes of a standard gelatin in a standard time is taken as a measure of the enzyme activity. Shwachman, Farber, and Maddocks (1943) substituted the film of gelatin on an $x$-ray film for tubes of gelatin. Such methods give a relatively crude measure of activity, e.g., 1, 2, 4, $8,16,32,64$ units, etc. Methods capable of greater precision include those which measure the decrease in viscosity of gelatin solutions during digestion, e.g., that of Northrop and Hussey (1923), and others which estimate quantitatively the products of protein breakdown. Among the latter are the methods of Ågren and Lagerlöf (1936) who titrated the carboxyl groups liberated from casein, and of Anson and Mirsky (1933) who measured the phenolic products of haemoglobin digestion colorimetrically.

We have used the method of Charney and Tomarelli (1947) which somewhat resembles the latter. It employs as substrate a coloured azoprotein formed by coupling diazotized aryl amines with proteins in alkaline solution. Digestion of this azoprotein complex by a proteolytic enzyme results in the liberation of coloured breakdown products, which remain in solution when trichloroacetic acid is added to precipitate any unchanged azoprotein. The intensity of the colour in the trichloroacetic acid filtrate of the digested substrate is therefore proportional to protein breakdown and provides a 2 measure of proteolytic activity. The test is made o fairly specific for trypsin by maintaining the $p \mathrm{H}$ at $\overparen{\Phi}$ $8 \cdot 3$, at which trypsin is highly active, whereas many $\stackrel{9}{+}$ other proteases, especially pepsin, have little activity.

The substrate used in the original method was sulphanilamide azocasein. By the procedure described, however, we were unable to obtain adequate diazotization, and the optical density of the final 
solution was much less than that quoted. We therefore adopted modifications suggested by Dr. J. Harkness (personal communication, 1953) that sulphanilic aoid be substituted for sulphanilamide, that the temperature of the reactants be kept below $4 \mathrm{C}$. while diazotisation and coupling were occurring, and that the solution be dialysed against water for 24 hours before precipitation with acetic acid and drying according to the original method.

\section{METHOD}

REAGENTS The following were used:-

1 Sulphanilic acid azocasein: Solution A Dissolve 50 g. re-precipitated and fat-free casein in $1,000 \mathrm{ml} .1 \%$ w/v aqueous sodium bicarbonate A.R. Cool to below $4^{\circ} \mathrm{C}$.

Solution $B$ Dissolve 5.0 g. sulphanilic acid A.R. in $200 \mathrm{ml}$. water containing $6.0 \mathrm{ml}$. $5 \mathrm{~N}$ sodium hydroxide A.R. Add 2.2 g. sodium nitrite A.R. and dissolve. Cool to below $4^{\circ} \mathrm{C}$. Add $18.0 \mathrm{ml}$. $5 \mathrm{~N}$ hydrochloric acid A.R. and stir for two minutes. Add $18.0 \mathrm{ml}$. $5 \mathrm{~N}$ sodium hydroxide A. R. and stir. Cool to below $4^{\circ} \mathrm{C}$.

Add the whole of cold solution B at once to cold solution A with stirring. Allow to stand for one hour below $4^{\circ} \mathrm{C}$.

Dialyse the solution against about 31 . distilled water for 24 hours at room temperature, using cellophane 300 , and changing the water repeatedly in the first few hours. Adjust the $p \mathrm{H}$ with glacial acetic acid A.R. to 4.5 by glass electrode to precipitate the azocasein.

Filter on a Buchner funnel and wash with approximately $500 \mathrm{ml}$. distilled water and approximately $500 \mathrm{ml}$. ethanol, and dry in air at room temperature.

2 Substrate Dissolve $2.5 \mathrm{~g}$. azoprotein in $50 \mathrm{ml}$. $1 \%$ sodium bicarbonate at $60^{\circ} \mathrm{C}$., with stirring. Cool. Adjust $p \mathrm{H}$ to 8.3 by glass electrode. Add water to $100 \mathrm{ml}$. This gives a concentration of $25 \mathrm{mg}$. $/ \mathrm{ml}$. Store at $0^{\circ} \mathrm{C}$. or below.

It is our practice to store volumes of substrate sufficient for one batch of tests in screw-capped bottles and keep them in a deep-freeze or the freezing compartment of a refrigerator.

$31 \% w / v$ sodium bicarbonate $A . R$.

$4 \quad 0.5 \% w / v$ sodium bicarbonate $A . R$.

$55 \% \mathrm{w} / \mathrm{v}$ trichloroacetic acid A.R.

$6 \quad 0.5 N$ sodium hydroxide A.R.

COLleCtion AND PRESERVATION OF SPECIMENS For duodenal samples a tube is passed, and after one hour its position is checked radiologically and adjusted if necessary. Fasting samples are collected without any stimulus to pancreatic secretion.

Specimens are brought to the laboratory immediately after collection. They are preserved in the freezing compartment of a refrigerator, and estimated on the same day. Samples of duodenal juice are examined for colour and reaction, and low values for duodenal trypsin are not accepted unless the sample is bile-stained and has a $p \mathrm{H}$ not less than $5 \cdot 5$.
DILUTION OF SPECIMENS We used the following methods.

Duodenal juice Add $0.5 \mathrm{ml}$. to $4.5 \mathrm{ml}$. of $0.5 \%$ sodium bicarbonate. Mix and add $0.5 \mathrm{ml}$. of mixture to $4.5 \mathrm{ml}$. of $0.5 \%$ sodium bicarbonate. This gives a final dilution of $1 / 100$.

Faeces Weigh accurately 4.5 to $5.5 \mathrm{~g}$. and add a volume of $0.5 \%$ sodium bicarbonate equal to nine times the weight of the faeces (1/10 dilution). Break up faeces thoroughly with a glass rod to form a suspension. Add $0.5 \mathrm{ml}$. of suspension to $4.5 \mathrm{ml}$. of $0.5 \%$ sodium bicarbonate giving a final dilution of $1 / 100$.

ESTIMATION Two tubes each containing $1.0 \mathrm{ml}$. of azocasein substrate are placed in a $37^{\circ} \mathrm{C}$. waterbath and warmed for about three minutes. For the test, $1.0 \mathrm{ml}$. of diluted specimen is added to one of the warmed tubes, and the time of addition is carefully noted. For a reagent blank, $1.0 \mathrm{ml}$. of $0.5 \%$ sodium bicarbonate is added to the second tube and again the time is noted. Each tube is incubated for exactly 30 minutes and the reaction stopped by the addition of $8.0 \mathrm{ml} .5 \%$ trichloroacetic acid, which precipitates the undigested azocasein. As the precipitate does not pack well when centrifuged at moderate speeds, it is removed by filtration using Whatman No. 1 paper.

To $5.0 \mathrm{ml}$. of filtrate is added $5.0 \mathrm{ml}$. of $0.5 \mathrm{~N}$ sodium hydroxide to develop the colour, giving a final substrate dilution of $1 / 20$. A substrate standard is prepared by taking $5.0 \mathrm{ml}$. of substrate diluted $1 / 200$ with water and adding $5.0 \mathrm{ml}$. of $0.5 \mathrm{~N} \mathrm{NaOH}$, so that the final dilution is $1 / 400$. This high dilution is necessary in order to get a reading on the photometer and the result must be multiplied by 20 to make it comparable with the $1 / 20$ dilution of substrate in the test. In a photometer with an Ilford filter 621 (maximum transmission $450 \mathrm{~m} \mu$ ) the test is read against the reagent blank, and the substrate standard against distilled water.

Calculation As in the original method, the unit of enzyme activity is based on the velocity constant. The enzymatic hydrolysis of protein is a first order reaction, the velocity constant $(\mathrm{k})$ of which is expressed by the equation:-

$$
\mathrm{k}=\frac{1}{\mathrm{t}} \times 2 \cdot 3 \log \frac{\mathrm{C}_{1}}{\mathrm{C}_{2}}
$$

where $t$ is the time of reaction in minutes (here standardized at 30 minutes) and $C_{1}$ and $C_{2}$ are initial and final substrate concentrations (here expressed in terms of optical density). The test is performed on a 1/100 dilution of the specimen so that:

$$
\begin{aligned}
& k=100 \times \frac{1}{30} \times 2.3 \log \frac{C_{1}}{C_{2}} \\
& k=7.67\left(\log C_{1}-\log C_{2}\right)
\end{aligned}
$$

As the value of $k$ is always less than 1 , the unit of activity is taken as $100 \mathrm{k}$ for convenience so that:

enzyme activity $=100 \mathrm{k}=767\left(\log C_{1}-\log C_{2}\right)$ where $\mathrm{C}_{1}=$ optical density of diluted substrate standard $\times 20$ (because previously diluted 20 times more than the substrate in the test), and

$$
\mathrm{C}_{2}=\mathrm{C}_{1} \text { - optical density of test. }
$$

Normal values for duodenal trypsin are about 10 to 60 units, and for faecal trypsin about 10 to 80 or more units. 


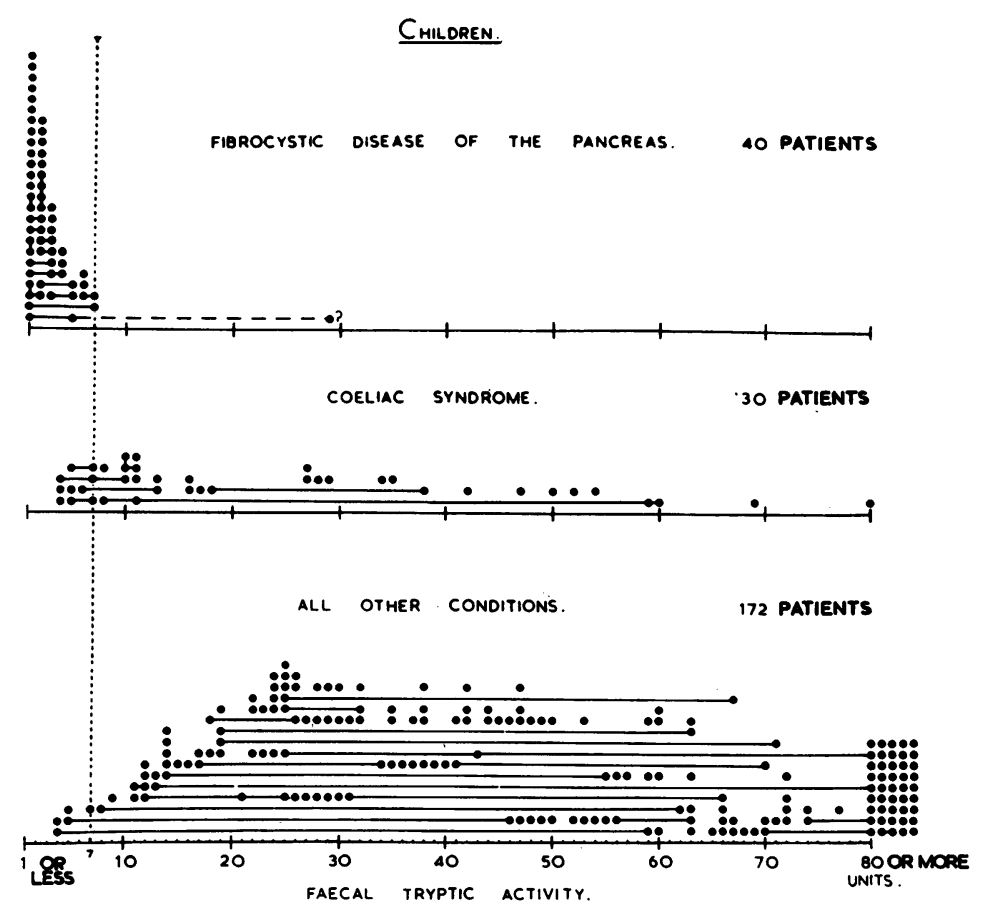

FIG. 1. Faecal tryptic activity in children suffering from fibrocystic 흐 disease of the pancreas (top), the coeliac syndrome (middle), and all $\underset{\mathbb{D}}{\vec{D}}$ other conditions (bottom). Each dot 0 represents a single estimation. Repeated estimations on the same patient $\overrightarrow{0}$ are joined by a line.

FIG. 2. Duodenal tryptic activity in children suffering from fibrocystic $\widehat{ }$ disease of the pancreas, the coeliac syndrome, and all other conditions. $\overrightarrow{ }$ Each dot represents a single est $-\overrightarrow{-}$ mation. Repeated estimations on the same patient are joined by a line.

FIG. 1

It is possible to measure levels above 80 units by repeating the estimation using less of the test sample, but as we were interested in low values only we have not done so.

\section{RESULTS}

To assess the diagnostic value of this test, the records of all patients who have had this test performed since its introduction into our laboartory in March 1953, have been examined. The final diagnoses of these

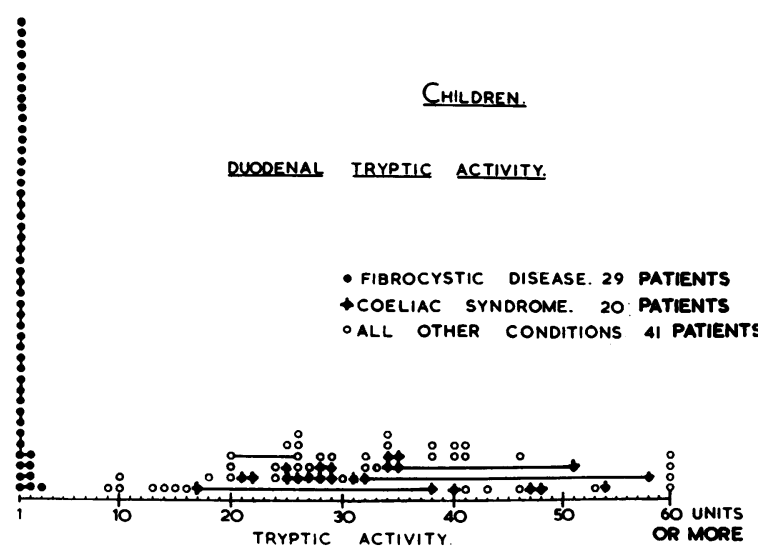

FIG. 2 patients have been correlated with the level of faecal trypsin.

For the purpose of discussion the patients have $\overrightarrow{\vec{O}}$ been divided into two age groups, consisting of 2423 children and 45 adults.

CHILDREN As this test has been used mainly in? children for the diagnosis of fibrocystic disease, the children are discussed first and are divided into two main subgroups: those with a final diagnosis of $O$ fibrocystic disease of the pancreas, and those with all other conditions.

Children with fibrocystic disease of the pancreas $\frac{D}{0}$ In this group there were 40 children, 39 of whom had faecal trypsin values of 7 units or less (Fig. 1). $N$ In 16 of these the estimation was repeated on more than one occasion with the same low value. In the one exception, a girl aged 1 year, three separate esti- $\omega$ mations of faecal trypsin gave values of 29 units, less than 1 unit, and 5 units over a period of six weeks; fibrocystic disease was proven at necropsy. Thus in only a single patient and in only one out of ${ }^{?}$ three estimations was the faecal trypsin found to be $\frac{T}{3}$ above 7 units. We consider that this could have been $\frac{\vec{D}}{\mathbb{D}}$ the result of a technical error in estimation, or of a $\odot$ mis-labelling of the specimen at the time of col- $\stackrel{\mathbb{Q}}{\Omega}$ lection; the patient was not taking pancreatin. 
In 29 of this group the duodenal trypsin also was estimated, and found to be 3 units or less in every case (Fig. 2). The patient mentioned above with one high faecal trypsin value was one of these shown to have a low duodenal trypsin level.

Children without fibrocystic disease of the pancreas It was found that low levels of faecal tryptic activity were not confined to patients with fibrocystic disease of the pancreas (Fig. 1). Of the remaining 202 children, 12 patients were found to have a faecal trypsin level of 7 units or less. Of these, seven patients belonged to a group of 30 with a final diagnosis of coeliac syndrome. All but one had successful duodenal intubation and were found to have duodenal trypsin levels of 17 units or more (Fig. 2).

Of the 172 patients without fibrocystic disease of the pancreas or coeliac syndrome, there were five with faecal trypsin levels of 7 units or less, but two of these patients on repeat estimations gave values of 59 and 46 respectively. In the other three only a single estimation was performed. Only one patient of these three had successful duodenal intubation, and the trypsin level was 20 units. There were therefore only two children with low faecal trypsin which might have been due to a deficiency of trypsin secretion; the final diagnoses in these patients were given as upper respiratory infection and otitis media, the faecal trypsin levels being 5 and 4 units respectively.

Of the remaining 167 patients, i.e., those without fibrocystic disease or coeliac disease and with normal trypsin levels, successful duodenal intubation was carried out on $\mathbf{4 1}$ and yielded duodenal trypsin levels of from 9 to over 60 units (Fig. 2).

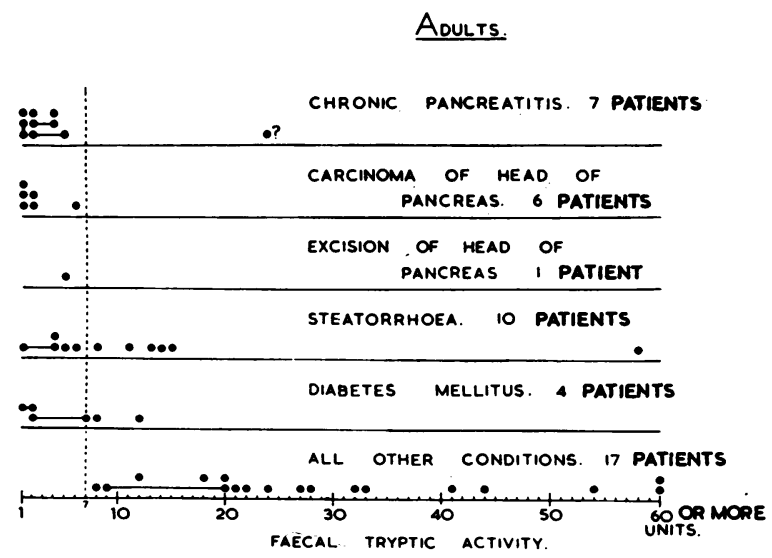

FIG. 3. Faecal tryptic activity in adults arranged according to diagnosis. Each dot represents a single estimation. Repeated estimations on the same patient are joined by $a$ line.
ADULTS Having found that children with fibrocystic disease nearly always had levels of faecal trypsin of 7 units or less, we then examined the records of the 45 adults on whom the test had been performed, and classified them according to faecal trypsin levels. It was found that those with a value of 7 units or less included patients with final diagnoses of chronic pancreatitis, carcinoma of the head of the pancreas, excision of the head of the pancreas, diabetes mellitus, and steatorrhoea (Fig. 3). The notes of all patients in this group with these diagnoses have been analysed.

Chronic pancreatitis of seven patients in this subgroup, six had faecal trypsin values of 5 units or less; one of these underwent duodenal intubation (the only adult to do so), and a duodenal trypsin level of less than 1 unit was found. The seventh patient was a woman aged 56 with a long history of vague upper abdominal pain, and a single faecal trypsin value of 24 units. The diagnosis was based apparently on one faecal fat estimation that gave a high unsplit fat level (total fat $32 \%$ of dried faeces, split $9 \%$, unsplit $23 \%$ ), although two previous estimations of faecal fat had given normal results; it could be that the abnormal result was due to the patient taking liquid paraffin. A glucose tolerance curve was flat. We feel therefore that the diagnosis was not established and that the patient should be excluded from this group.

Carcinoma of the head of the pancreas Of six patients in this subgroup all had faecal trypsin values of less than 6 units.

Excision of the head of the pancreas There was only one such patient, who had a faecal trypsin level of 5 units.

Diabetes mellitus There were four such patients, of whom two had faecal trypsin values of 7 units or less on more than one occasion and both had steatorrhoea. In the remaining two patients, values of 12 and 8 units respectively were obtained and were not repeated; the former had steatorrhoea, and the other had no faecal fat estimations.

Idiopathic steatorrhoea Out of 10 such patients, four had faecal trypsin values of 7 units or less; three of these four had flat glucose tolerance curves.

Other conditions In all 17 patients with diseases other than those classified above, faecal trypsin values were more than 7 units.

\section{DISCUSSION}

The value of faecal trypsin estimation in the diagnosis of pancreatic disease depends upon (1) the extent to which duodenal trypsin levels reflect pancreatic disease, and (2) the extent to which faecal trypsin levels reflect duodenal trypsin levels. 
1 There is little doubt that in children with fibrocystic disease duodenal trypsin levels are in general very low in comparison with other children. Thus Bodian (1952) using Andersen and Early's method (1942) found that in 79 out of 80 cases of fibrocystic disease digestion occurred only at dilution less than $1 / 25$ (the exception having minimal pancreatic lesions at necropsy), whereas in 294 out of 296 controls digestion occurred at levels of 1/50 or more. Payne (1952), who used the same method and whose cases are probably included among those of Bodian, reported that in 98 out of 100 controls digestion occurred at $1 / 50$ dilution or more, whereas in 25 cases of fibrocystic disease no digestion occurred above $1 / 12.5$ dilution. In our series all 29 cases of fibrocystic disease had duodenal trypsin levels of 3 units or less while 61 controls had levels of 9 units or more. However, since the introduction of the sweat test (di Sant'Agnese, Darling, Perera, and Shea, 1953), incomplete cases have been diagnosed in which pancreatic function has been normal or only moderately impaired. Thus Shwachman and Kulczycki (1958) mention that in 21 out of 105 fibrocystic patients the initial duodenal fluid sample showed 'partial pancreatic insufficiency or normal enzyme activity', although in five of these 'progression to complete loss of pancreatic enzyme activity was noted'. Similarly di Sant'Agnese (1959), who used a viscosimetric method of trypsin estimation, made the diagnosis in the absence of severe pancreatic dysfunction in 34 out of a total of 243 cases, only two out of 254 cases having normal sweat electrolytes. If then severe pancreatic deficiency is not constantly present in fibrocystic disease, a deficiency of trypsin secretion is no longer necessary for the diagnosis, but remains a useful test of pancreatic involvement.

In adult patients most investigators of pancreatic disease have reported duodenal trypsin levels only after stimulation with secretin and, more recently, pancreozymin, e.g., Marks and Tompsett (1958), and there is little information concerning resting levels. Voegtlin, Greengard, and Ivy (1934), using a different casein digestion method, reported normal resting values $(0.25$ to 2.7 units) but no figures for pancreatic disease. Ågren and Lagerlöf (1936), using the same method, reported output per minute and not concentrations, and as the volume secreted was very variable no conclusions as to concentrations can be drawn. Dornberger, Comfort, Wollaeger, and Power (1948) investigated duodenal juice before and after secretin. However, the data before secretin were 'not presented in detail. The results may be summarized by saying that average values for some of the determinations were lower for the group of patients with pancreatitis than for normal persons, but the values for normal persons ranged so low that only when almost complete 은 absence of bicarbonate and of enzymes occurred $\vec{F}$ did the data confirm the known existence of extensive parenchymatous destruction'. It is not clear whether this refers to concentrations or outputs of enzymes. Our own experience of duodenal trypsin levels in adults is limited to a single patient with chronic pancreatitis in whom the level was very low.

It may then be concluded that in fibrocystic $\overrightarrow{0}$ disease duodenal trypsin levels are as a rule significantly lower than those of controls but that in a $\vec{\omega}$ few cases there may be little pancreatic involvement and normal enzyme levels. In adults it is likely that ? duodenal trypsin levels will be low in severe pan- $\vec{G}$ creatic disease, but there is insufficient information concerning normal levels to be sure of the diagnostic ${ }_{\mathrm{N}}$ value of such a result in the absence of hormonal 을 stimulation of the gland.

2 It is generally accepted that the correlation $c$ between duodenal and faecal trypsin levels is not close (Howat, 1958). In children, Shwachman, Patterson, and Laguna (1949), using the relatively crude gelatin film method, estimated faecal trypsin $\vec{\varnothing}$ levels in $\mathbf{5 0 0}$ healthy subjects and patients 'exclusive ? of pancreatic fibrosis'. They found negative values $\square$ (no perceptible digestion at $1 / 5$ and $1 / 10$ dilution after one hour's incubation at $37^{\circ} \mathrm{C}$.) in $4 \%$ of 74 subjects between 5 and 10 years, and in $75 \%$ of $32 \%$ over 10 years. It is to be doubted whether all these $\mathbb{D}$ patients had very low duodenal trypsin levels. $\stackrel{\varrho}{\Rightarrow}$ Similarly Payne (1952), who used a tube-gelatin 응 method, found that in children without pancreatic disease and with normal duodenal trypsin levels the faecal trypsin might be very low, and that this was common over the age of 2 years but rare under 1 year. Bodian (1952), whose cases probably included those 0 of Payne, found that those without pancreatic disease 3 . usually had normal duodenal levels, whereas nearly $\delta$ half had low faecal levels.

Nevertheless both Payne and Bodian found that $\mathrm{O}$ the low duodenal trypsin levels found in all but one $\square$ of their cases of fibrocystic disease were associated with low faecal trypsin levels whenever both were estimated in the same case.

Our results confirm that cases of fibrocystic disease $N$ with low duodenal trypsin levels all have low faecal స్ట trypsin levels. They also confirm that in children low faecal levels may coexist with normal duodenal levels, particularly in the coeliac syndrome. There is, $\frac{\mathscr{D}}{\mathbb{D}}$ however, very much less overlap between faecal $\stackrel{\infty}{?}$ trypsin levels found in patients with fibrocystic 0 disease and those found in controls than was found 0 by the authors mentioned above; if the coeliac $\stackrel{\mathbb{D}}{\Omega}$ syndrome is excluded, only five of 172 children had $\mathbb{\Phi}$ levels as low as those with fibrocystic disease. This $\frac{\varrho}{\sigma}$ 


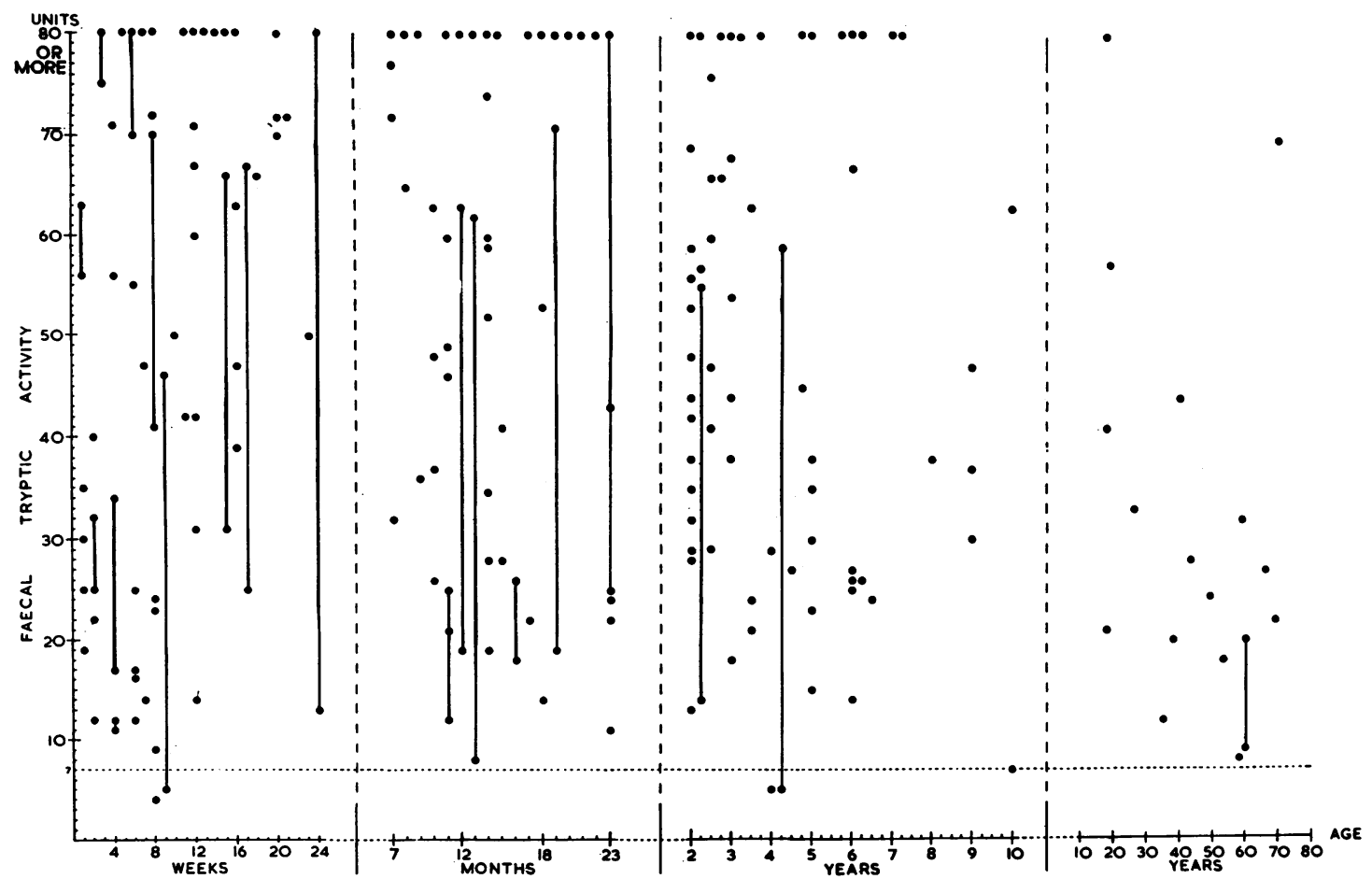

FIG. 4. Faecal tryptic activity plotted against age for 172 children (excluding those with fibrocystic disease and the coeliac syndrome) and 17 adults (excluding those with pancreatitis, carcinoma of the pancreas, excision of the head of the pancreas, steatorrhoea, and diabetes mellitus). Each dot represents a single estimation. Repeated estimations on the same patient are joined by a line.

may be due, in part at least, to the method of estimation which we used, because it allows trypsin levels to be measured with greater precision than the older doubling dilution methods. This group of 172 children varied in age from a few days to 10 years, but there is no obvious correlation between trypsin level and age (Fig. 4); above 10 years, levels may be rather lower. This finding, so different from those of Shwachman et al. (1949) and Payne (1952), suggests that the results obtained by gelatin digestion and by azocasein digestion may not be strictly comparable.

In adults absence of duodenal trypsin estimations in all but one of our cases makes correlation with faecal levels impossible. It is very likely, however, that the relationship is similar to that found in children, for low faecal levels are found not only in those cases in which there is gross pancreatic disease, but also in others without obvious disease of the gland in whom it is probable that duodenal trypsin levels are normal, e.g., in idiopathic steatorrhoea.

The reason for low faecal trypsin levels in the presence of normal duodenal levels has not yet been ascertained, but it is much commoner in the presence of steatorrhoea, particularly in coeliac disease in children (seven out of 30 cases) and probably in adult cases of steatorrhoea. It could be due to destruction of the enzyme during passage down the gut, or to the presence of inhibitors in the faeces.

The finding of low faecal trypsin levels in diabetes mellitus is interesting and requires further investigation. It may be related to steatorrhoea, which is known to have been present in at least three of the four cases investigated, but it may be a sign, in a proportion of such cases, that the diabetes is due to generalized disease of the pancreas.

\section{CONCLUSIONS}

From these results, it appears that patients with severe impairment of exocrine pancreatic secretion have consistently low faecal trypsin levels as well as low duodenal trypsin levels. Low faecal trypsin levels, however, do not necessarily indicate pancreatic insufficiency; for instance, in coeliac disease it is common to find low faecal but normal duodenal trypsin levels, and the same is probably true of idiopathic steatorrhoea in adults. 
The estimation of faecal trypsin levels cannot therefore be used as a specific test of pancreatic disease, but it does appear to provide a useful screening test for the selection of those patients who might have chronic pancreatic disease and in whom it is worth while carrying out further more complex investigations such as the estimation of duodenal tryptic activity.

So far we have encountered no convincing exception to the rule that patients with a low duodenal trypsin will have a consistently low faecal trypsin, but our direct evidence on this point is virtually confined to children.

We thank the consultant paediatricians, physicians, and surgeons of the United Bristol Hospitals for the opportunity to study the patients under their care.

\section{REFERENCES}

Agren, G., and Lagerlöf, H. (1936). Acta med. scand., 90, 1. Andersen, D. H., and Early, M. V. (1942). Amer. J. Dis. Child., 63, 891.

Anson, M. L., and Mirsky, A. E. (1933). J. gen. Physiol., 17, 151.

Bodian, M. (1952). Fibrocystic Disease of the Pancreas. Heinemann, London.

Charney, J., and Tomarelli, R. M. (1947). J. biol. Chem., 171, 501. di Sant'Agnese, P. A. (1959). Pediatrics, 24, 313.

- Darling, R. C., Perera, G. A and Shea, E.(1953). lbid., 12, 549.

Dornberger, G. R., Comfort, M. W., Wollaeger, E. E., and Power, M. H. (1948). Gastroenterology, 11, 701.

Howat, H. T. (1958). In Modern Trends in Gastroenterology (second series), edited by F. Avery Jones, p. 401. Butterworth, London.

Marks, I. N., and Tompsett, S. L. (1958). Quart. J. Med., n.s. 27, 431.

Northrop, J. H., and Hussey, R. G. (1923). J. gen. Physiol., 5, 353.

Payne, W. W. (1952). Gt Ormond Str. J., No. 3, p. 23.

Shwachman, H., Farber, S., and Maddocks, C. L. (1943). Amer. J. Dis. Child., 66, 418.

and Kulczycki, L. L. (1958). Ibid., 96, 6.

, Patterson, P. R., and Laguna, J. (1949). Pediatrics, 4, 222.

Voegtlin, W. L., Greengard, H., and Ivy, A. C. (1934). Amer. J. Physinl., 110, 198. 